\title{
An Evaluation of Adaptation Options to Climate Pressure on Highland Robusta Coffee Production, Daklak Province, Vietnam
}

\author{
Thuyen Pham Thi ${ }^{1,2, *}$, Yaovarate Chaovanapoonphol ${ }^{3}$ \\ ${ }^{1}$ Center for Agricultural Resource System Research, Faculty of Agriculture, Chiang Mai University, Chiang Mai, Thailand \\ ${ }^{2}$ Department of Economic, Faculty of Economic, Nong Lam University, Ho Chi Minh City, Vietnam \\ ${ }^{3}$ Department of Agricultural Economics and Extension, Faculty of Agriculture, Chiang Mai University, Chiang Mai, Thailand \\ *Corresponding author: phamthuyen@hcmuaf.edu.vn
}

Received August 27, 2014; Revised September 01, 2014; Accepted September 10, 2014

\begin{abstract}
Adaptation to changes of temperature and rainfall is a two-stage process, which initially hinges on the farmers' perception of climate variability and then responding to changes through adaptation strategies. Adaptation evaluation is considered as part of a planned policy coping with consciously planned, primarily anticipatory adaptation initiatives undertaken by decision makers, specifically individual farmers. An evaluation goes beyond the identification, characterization of adaptation approaches and with regards to an adaptation option's relative merit, superiority or implement-ability. Evaluative criteria do not only mention on principally economic dimension, but also relate to the different considerations. The objectives of this study (1) undertakes an evaluation of adaptation options in level of coffee farms by five alternatives involving in effectiveness, economic efficiency, flexibility, farmer implement-ability and independent benefits and (2) analyzes the determining factors impacting on the farmers' adaptation level. The study uses data from structured interviews with 176 coffee farmers in Ea H'leo District, Daklak Province, Vietnam. The multiple criteria evaluation, unity based normalization and weighted sum methods are employed to assess the farmers' adaptation options. The Ordered logit model is also used to estimate the relationship between the farmers' adaptation level and their demographic and socio-economic characteristics. The result of multiple criteria evaluation indicated that amongst five evaluative criteria, the economic efficiency and effectiveness were assessed with the highest weights about importance level. The outcome of weighted sum of adaptation options highlighted that the level of adaptation was not positive relationship with the number of adaptation options which the farmers adapted to climate pressure for their coffee farm. It depended on the adaptation's multiple considerations. The findings of regression model also revealed that factors related to the households' socio-economic characteristics had statistically significant impacted to choosing the adaptation level at significant level 1\%, $5 \%$.
\end{abstract}

Keywords: climate change, adaptation evaluation, robusta coffee, Vietnam

Cite This Article: Thuyen Pham Thi, and Yaovarate Chaovanapoonphol, "An Evaluation of Adaptation Options to Climate Pressure on Highland Robusta Coffee Production, Daklak Province, Vietnam.” World Journal of Agricultural Research vol. 2, no. 5 (2014): 205-215. doi: 10.12691/wjar-2-5-2.

\section{Introduction}

Climate change is currently highlighted in the international community as a potential threat to coffee production in the Central highland environments of Vietnam. It has already impacted on innumerable communities, exposing them to increasing hazards and making them more vulnerable [1]. Dak Lak where is the largest province of Vietnam, located in the Central Highland with the total area of 1,312,537 ha, including 480,000 hectares of agricultural land [2]. Under the weather condition of the Central Highland, Daklak is very suitable for high value industrial crops cultivating such as coffee, peper and rubber. However, being a hilly area that does not hold impounded surface water and also is the area with moderate tropical climate, Dak Lak is seriously affected by high evaporability from climatic variability; the demand for irrigation in agriculture is calculated to be two-to threefold compare to current demand [3]. Temperature and rainfall conditions are considered to be important factors in defining potential coffee yield. The coffee plant responds sensitively to increasing temperatures, specifically during blossoming and fructification [4]. The high temperature results in increasing water demand for crops while water resource is becoming scarce. Coffee is a water-intensive perennial crop. The groundwater is the major source for coffee irrigation, using up about 66 million $\mathrm{m}^{3}$ during the dry season, or $438,400 \mathrm{~m}^{3}$ per day. In the dry season, in the blossom and fruit period, around 1,500-3,000 $\mathrm{m}^{3}$ of water per hectares need to be provided for coffee [5]. According 
to latest press releases the coffee sector is already suffering from climate variability as the 2010-2011 harvest output is expected to decline by $20 \%$ compared with the previous harvest due to extreme drought period and delayed rainfalls. The coffee productivity, reduced from 3.5 t/ha to 2t/ha/year [6]. Also owning to extreme drought, the area of growing coffee in Ea H'leo District, Daklak province is damaged about 2,925.71 ha whereas, the level of damage less than $30 \%$ is $1,318.09$ ha and more than 30\% with 1,607.62 ha in 2012 - 2013 [7].

Climate pressure, such as shifts in the rainy season and variations in temperature and precipitation can adversely affect coffee plant physiology, resulting in reduced yields. Some analyses of climate pressure impacts in the region anticipate that certain coffee-growing regions will face on rising temperatures or changed climate patterns that may render production unprofitable or infeasible [8]. For smallholder farmers, the move to quality production, including quality related certifications, requires access to information and technical assistance, which can be difficult and require substantial investments of time and labor. The mode of disseminating agricultural policies and programs, which govern access to technical support and services as well as credit and knowledge, poses part of the challenge. Promoting sustainable development in the uplands of the Central Highland poses important challenges. Thus, it is essential to include specific and planned adaptation strategies to climate pressure. And adaptation practices require extensive high quality data and clear information on climate, agricultural, environmental and social systems which support considerably for assessing impacts of climate variability.

In order to determine which adaptations should be promoted or implemented adaptation options need to be evaluated. The assessment needs to reflect the overall merit of alternative adaptation options to identify appropriate adaptation approaches [9,10].

Famers' evaluation of private adaptive options depending on their perceive self-efficacy ability and subjective for the evaluative alternatives and adaptive measures. Climate change includes rapid changes in climatic variables such as temperature, radiation and precipitation as well as changes in the atmospheric concentration of greenhouse gases, soil water and nutrient cycling. It is not easy to measure the level of the respondent's perception and adaptation to climate change in general; therefore, the study will focus on the change of temperature and rainfall in the region.

Using data from structured interviews with 176 coffee farmers in Ea H'leo District, Daklak Province, Vietnam, the study investigates how coffee farmers evaluate their private adaptive approaches to climate pressure. And analyzing the factors' impact on farmers' adaption level is also undertaken in this research.

\section{Materials and Methods}

\subsection{Materials}

The domain of study was carried out in Ea H'leo district, where a certain group of farmers was impacted by climate variability and based on coffee production. The main criteria for selecting of this district were that it had a heavy concentration of coffee area. Coffee was a dominant crop and considered as a backbone of household's income and livelihood. Almost all the households were both directly and indirectly engaged in coffee production. Moreover, the frequency and severity of extreme drought in the region have been increasing and declined rainfall in recent years. This causes challenges for coffee sustainability in this area.

A simple random sampling method was adopted to select households for questionnaire survey. The sample size for farmers is calculated based on Yamane's formula [11].

$$
n=\frac{N}{1+N^{*} e^{2}}
$$

Where, $\mathrm{n}$ is the sample size

$\mathrm{N}$ is the size of coffee farming population

e is allowable error $7.5 \%$

By using this formula, with total coffee farming in the study area is 23,613 households [12] and due to the time and cost limitation, the study accepts the allowable error $7.5 \%$ instead of error $5 \%$. With the result that, interview consists 176 farmers in the selected villages belong to Ea H’leo district, in Dak Lak province.

\subsection{Methods}

\subsubsection{Multiple criteria Evaluation}

Adaptation refers to responses of individuals to climatic effects in order to reduce vulnerability or unfavorable impacts or damage potential. Evaluation of the adaptation options is intended to assess the overall merit, suitability, utility or appropriateness of potential adaptation strategies or measures $[13,14,15,16]$.

The evaluation framework was designed by the Intergovernmental Panel on Climate Change [14], along with others developed under its framework $[15,17,18]$ suggested various methodologies for the evaluation of adaptation options in decision-making coping with climate change. These included benefit-cost analysis, cost effectiveness analysis, risk benefit analysis, multiple objective analyses, and multiple criteria evaluation. In this study, multiple criteria evaluation (MCE) was selected for evaluation because assessment alternatives used more than one criterion $[15,19,20]$, not only on principally economic factors. MCE allowed consideration of both quantitative and qualitative data in the ranking of alternative options. Its approaches included identifying alternative options, select criteria, scores (weighted scales) options against criteria, assign weights to each criterion, calculate weighted sum and rank options [21].

There are various researches, providing the evaluative criteria for assessing adaptation options under different scales. In level of the farm, effectiveness was often considered as a first step in adaptation evaluation. [15] illustrated the utility of identifying the effectiveness of various adaptation options in meeting specific objectives under alternative climate change scenarios. It refers to the ability of the adaptation option to reduce the income loss as a result of increases in frequency and magnitude of the temperature and rainfall changes. A very effective adaptation option will eliminate the risk of income loss, while an ineffective adaptation option will not greatly 
change this risk. Therefore, those options that eliminate the risk of income loss are more desirable and will receive a higher score than those that do not reduce risk. The evaluations can be converted to five categories of effectiveness ( 1 = very ineffective, 2 = ineffective, $3=$ neutral, 4 = moderately effective, 5 = very effective). In order to illustrate the conversion from a monetary scale, 'moderately effective' could be defined where a measure averts between $20 \%$ and $50 \%$ of expected income loss, whereas 'very effective' averts greater than $50 \%$ of loss [15].

Economic efficiency is also as an important criterion in the adaptation evaluation literature. It refers to the economic benefits of the adaptation relative to the economic costs of implementing the adaptation option. Economic efficiency is commonly measured as the ratio of benefits to costs. The value of this ratio can be used to establish levels of efficiency and represented in the Likert scale. For example, an adaptation option, which has a benefit-cost ratio greater than 1.5 may be designed as very economically efficient, ratios between 1.1 and 1.5 are moderately efficient, ratios between 1 and 0.9 are of neutral efficiency, ratios between 0.9 and 0.8 inefficient, and ratios less than 0.8 are very inefficient $(1=$ very inefficient, 2 = moderately inefficient, $3=$ neutral, $4=$ moderately efficient, 5 = very efficient) $[9,22]$.

[9] identified evaluative criteria, including flexibility in their evaluation of adaptation options for climate-sensitive sectors in Africa. Evaluative criteria suggested by [13] also included flexibility (the ability of an adaptation to perform well for a range of likely climate changes). Similarly, in their evaluation of agricultural adaptation options in Kazakhstan, [22] identified flexibility (options meet policy objectives under a wide range of climate change conditions) as one essential criterion in identifying anticipatory adaptation options. Flexibility refers to the ability of the adaptation option to function under a variety of climate change conditions. For example, a very flexible adaptation option will avert income loss, whether the frequency of drought increases by $0 \%, 5 \%, 20 \%, 50 \%$ or $100 \%$, and whether there are changes in the magnitude, timing or duration of climate pressure, and perhaps associated heat stress or other related problems. An adaptation option that will only reduce income loss under a very particular set of climate conditions, and is ineffectual for other climate change conditions, is considered to be inflexible. Measurement of flexibility could be based on formal probability assessment, such as the ability to deal with specified drought frequency regimes, a decreasing trend in the annual amount of rainfall, a delay in the onset of rainy season and their associated risks. In this analysis, flexibility is measured directly on the five point Likert scale ( 1 = very inflexible, 2 = moderately inflexible, $3=$ neutral, $4=$ moderately flexible, 5 = very flexible).

$[9,23]$ agreed that prevailing uncertainties constrain the identification, assessment and implementation of adaptation options. The decision-making environment in agriculture was complex and the implementation of an adaptation option in an often highly specialized production system was not always straightforward and simple [24,25]. An adaptation option can be implemented by a farmer given existing management, established practices, farmer values and resources. In this study, an adaptation option that has a high degree of understandability, observability and compatibility with operations is considered to have a high degree of farmer implement-ability which is considered as an indispensable criterion for adaptation evaluation $(1=$ very low implement-ability, 2 = moderately low implement-ability, 3 = neutral, 4 = moderate implement-ability, 5 = very high implement-ability). Those that have a high degree of complexity, and are not socially and culturally acceptable, and/or do not fit readily with established management practices, investment strategies or technology are considered to have very low farmer implement ability.

Independent benefits that refer to the ability of an adaptation option to generate benefits independent of climate change, is also an important evaluative criterion. The adaptation options that reduce the risk of income loss regardless of climate change are more desirable to farmers than the options that are helpful only in addressing climate change risks, or that require some kind of trade-off (1 = high tradeoffs, 2 = moderate tradeoffs, $3=$ neutral, $4=$ moderate independent benefits, $5=$ high independent benefits) [9]. An adaptation strategy is viewed more favorably, the greater the benefits it brings, quite apart from its contribution to reducing or avoiding risk associated with climate change.

\subsubsection{Unity Based Normalization and Weighted Sum Method}

After using five alternatives in order to evaluate the adaptation options, the study used the unity based normalization method (features scale) to make the categories of criteria to scale the range in $[0,1]$ for each adaptation option. The general formula [26] was given as:

$$
x^{\prime}=\frac{x-\min (x)}{\max (x)-\min (x)}
$$

Whereas $\mathrm{x}$ was an original value (the value which farmers responded the scale for each adaptation appropriately each criterion), x' was the normalized value. Then, the study used the weight method [27] to obtain the weighted scale for adaptation option in proportion to each criterion. The weighted scale for each adaptation was computed as:

$$
W_{j}=\frac{\sum S_{i}}{\sum n_{j}}
$$

Whereas, $\mathrm{W}$ is the weighted scale corresponding each adaptation; $S$ is the value of five points which farmer i (1, n) responds for each adaptation in proportion to each criterion; $\mathrm{n}$ is the number of farmers who adjust to adaptation $\mathrm{j}$.

The results of the evaluation are shown using the MCE aggregation method. A subsequent evaluative step can be undertaken where each selected criterion is explicitly assigned a weight according to its significance or importance relative to the other criteria. The assigning of weights to each criterion is a subjective exercise and can be completed in a variety of ways. It may be assigned by expert panels, public participation process, researchers or government decision makers [28]. In this study, the weighted was assigned by individual farmers; they assigned their own weights, reflecting their personal 
values, goals and expectations. The study used Likert rating scale with 5 points as $1=$ very low importance, $2=$ low importance, $3=$ medium, $4=$ importance, $5=$ very importance for representing the importance level of each criterion in adaptation evaluation. And the unity based normalization method was also used to assign the weight for each criterion under scale ranging in $[0,1]$.

The next step, the weighted scale of each adaptation was multiplied by the assigned criterion weight before the values are summed to establish a single evaluative measure for each adaptation option. The weighted sum [29] was given as:

$$
F_{j}=\sum_{i=1}^{k} W_{i} \cdot W_{j}
$$

with $F_{j}$ is the weighted sum of each adaptation, $W_{i}$ is the assigned weights of the criterion $\mathrm{i}, \mathrm{W}_{\mathrm{j}}$ is weighted scale of adaptation $\mathrm{j}$, $\mathrm{k}$ is the number of evaluative criteria.

Using the equal interval scale method [30] ranked the adaptation options in three different adaptation categories as high, medium and low:

$$
I S=\frac{H V-L V}{N}
$$

whereas, IS is interval scale, $\mathrm{HV}$ is the highest value, $\mathrm{LV}$ is the lowest value and $\mathrm{N}$ is number of classes.

\subsubsection{Ordered logit model}

After using the multiple criteria evaluation tool for adaptation evaluation, the ordered logit model is employed to analyze factors impacting on the farmers' choice of adaptation categories adopted to mitigate climate pressure effects in the study area. The dependent variable is ordinal adaptation categories as high $=2$, moderate $=1$ and low $=$ 0 derived through the Multi-Criteria Evaluation tools with five criteria, including effectiveness, economic efficiency, flexible, farmer implement ability and independent benefit above.

Ordinal logit model is built around a latent regression in the same manner as the binomial choice model.

$$
y^{*}=\beta^{\prime} X_{i}+\varepsilon
$$

Where $\mathrm{y}^{*}$ is the underlying latent variable that indexes the level of contributions of respondents to perception decision making, $\mathrm{X}$ is a vector of parameters to be estimated, $\varepsilon$ is the error term.

The latent variable exhibits itself in ordinal categories, which could be coded as $0,1,2$. And its framework can then be used to form a model:

$\mathrm{Y}=0$, low adaptation if $\mathrm{y}^{*} \leq 0$

$=1$, moderate adaptation if $0<y^{*} \leq \delta_{1}$

$=2$, high adaptation if $\mathrm{y}^{*}>\delta_{1}$

with the $\delta$ 's being threshold parameter to be estimated with $\beta$ [31]. $\mathrm{X}_{\mathrm{i}}$ represents a set of conditioning variables which are the household attributes like:

$\mathrm{X}_{1}$ : Age of head of household (years)

$\mathrm{X}_{2}$ : Gender of head of household ( 1 for female, 0 for male)

$\mathrm{X}_{3}$ : Education level of head of household (years)

$\mathrm{X}_{4}$ : Coffee cultivation experience (years)

$\mathrm{X}_{5}$ : Coffee farming size (ha)

$\mathrm{X}_{6}$ : Coffee growing income $(1,000,000 \mathrm{VND} / \mathrm{year})$
$\mathrm{X}_{7}$ : Non-coffee income(1,000,000VND/year)

$\mathrm{X}_{8}$ : Access to credit ( 1 for accessing, 0 for otherwise)

$\mathrm{X}_{9}$ : Access to climate information (1 for accessing, 0 for otherwise)

$\mathrm{X}_{10}$ : Access to extension services ( 1 for accessing, 0 for otherwise)

$\mathrm{X}_{11}$ : Irrigation option (1 for irrigation option, 0 for otherwise)

The expected relationship between explanatory and dependent variables:

Age can be a factor determining individuals' differences because age relates to past experiences which make them have wider maturity and thought. A person in different age would have different knowledge and capability as well as experience. The age is positive impact with choosing of adaptation options.

Gender: Male-headed households are more likely to get information about new technologies and undertake risky businesses than female-headed households [32]. Moreover, [33] argued that having a female head of household might have negative effects on the adoption of soil and water conservation measures, because women might have limited access to information, land, and other resources due to traditional social barriers. So the research also aims to explore the choosing of adaptation and gender relationship.

Education is a basic factor leading to the individual's different perception and adaptation. Education helps people to increase their perception and understanding about climate variability and impacts as well as practicing adaptation approaches for their coffee farming. Higher level of education is believed to be associated with access to information on improved technologies and higher productivity [34]. Evidence from various sources indicates that there is a positive relationship between the education level of the household head and the adoption of improved technologies [35] and adaptation to climate change [36]. Therefore, the farmers with higher levels of education are more likely to adapt better to climate change.

Coffee cultivation experience is another factor directly affecting on adaptation. Experienced farmers have high skills in farming techniques and management and are able to spread risk when facing climate variability. The majority of the respondents are matured and more experienced in farming, and assumed to have a better knowledge and information on changes in climatic conditions as reported by [37]. There are a positive relationship between experience and choosing adaptation.

Coffee farming size: Farm size is always associated with greater wealth rather than capital and resources, the larger the farmer's farm size, the more likely the probability of adapting to climatic change in the study area.

Coffee growing income and Non-coffee income represent wealth. It is regularly hypothesized that the adoption of agricultural technologies requires sufficient financial wellbeing [38]. Other studies that investigate the impact of income on adoption found a positive correlation [39]. Higher-income farmers may be less risk averse and have more access to information, a lower discount rate, and a longer-term planning horizon [40].

Access to credit: Availability of credit eases the cash constraints and allows farmers to buy purchased inputs such as fertilizer, improved crop varieties, and irrigation 
facilities in order to reduce the negative impacts of climate change. Research on adoption of agricultural technologies indicates that there is the positive relationship between the level of adoption and the availability of credit [41,42].

Access to climate information: There is the same impact relationship as accessing to extension services. Accessing to climate information plays a vital role in improving the knowledge and awareness. If the farmers access to the information, they will perceive and have appropriate solutions for their farm under the adverse effects of climate pressure.

Access to extension services: Having access to extension services increases the likelihood of using adaptation options mentioned above in the study area. This study is in line with various studies in developing countries that report a positive relationship between access to information and the adoption behavior of farmers [41], and that access to information through extension increases the likelihood of adapting to climate change [36,37].

Irrigation option: Under changes of temperature and rainfall, water irrigation is scarcity and makes high pressure for the coffee farming. Households with access to irrigation system, they reduce risk, pressure for irrigating and meet water demand for coffee resulting in enhancement of coffee productivity. Therefore, they have the capability to adapt negative impacts of climate change.

\section{The Results}

The adaptation strategies were evaluated by five criteria through the multiple criteria evaluation tool and ranked under three-point scales. The dependent variable was ordered and categorical. The study estimated the effect of the determining factors on the different adaptation levels by an ordered logit model, with various possible explanatory variables related to socio-economic characteristics of coffee households.

\subsection{Multiple Criteria Evaluation Adaptation Options}

\subsubsection{Assigning Weights to Evaluative Criteria}

With a five-point scales of evaluative criteria ( 1 = very low importance, 2 = low importance, 3 = medium, 4 = importance, $5=$ very importance), the finding of interviewing 176 farmers about their response for importance level of each criterion in adaptation evaluation indicated that most of farmers answered the importance level of evaluative criteria from medium to very high. There were around $73.9 \%$ and $97.1 \%$ farmers who responded the effectiveness, economic efficiency was very importance level (5) respectively. The remaining percentage responded in the importance level (4). Meanwhile, the flexible and independent benefits were evaluated in medium importance level (3) with 68\% proportionally; 32\% responded were important (4) for two of these criteria. In term of implement-ability, there were $56.3 \%$ farmers presented that it was considered as an important indicator (4) and the medium level (3) took up about $43.7 \%$.

Hinging on the farmers' responses under five scales in proportion to each criterion, the study used unity based normalization method to rescale into the range in $[0,1]$. After calculating the weight of scales, the result was represented in Table 1 . The outcome exposed that amongst five criteria, the economic efficiency was assessed very importance with the highest weight 0.98 , following the effectiveness criterion with 0.95 . This was also synonymous with the importance level of two these criteria was nearly equal, played essential role for adaptation evaluation and farmers also mentioned more to capacity of reducing income loss of adaptation options. The weight of farmer implement-ability alternative was the third with 0.64 while flexibility and independent benefits were assigned the equal weight 0.58 respectively.

\begin{tabular}{cc} 
Table 1. Criterion Weighting & \\
\hline Criterion & Weight \\
\hline Economic efficiency & 0.98 \\
Effectiveness & 0.95 \\
Farmer Implement-ability & 0.64 \\
Flexibility & 0.58 \\
Independent benefits & 0.58 \\
\hline
\end{tabular}

Source: survey, 2013

\subsubsection{Assigning Weights to Evaluation of Adaptation Options}

Facing on changes in temperature and rainfall, the coffee growers selected the adaptation options (Table 2) for their farm in order to cope with climate pressure. In the total 176 farmers, there were 101 farmers adjusted to one adaptation option; 54 respondents adapted to two options and 21 remaining farmers acclimatized to three options.

Table 2. The Adaptation Options in Proportion to Number of Farmers

\begin{tabular}{cccccccc}
\hline Adaptation & A & B & C & AB & AC & BC & ABC \\
\hline Number of farmers & 30 & 40 & 31 & 15 & 15 & 24 & 21 \\
\hline
\end{tabular}

Note: A - Crop diversification, B - Irrigation techniques, C - Soil conservation, AB - Crop diversification and irrigation techniques, AC - Crop diversification and soil conservation, BC - Irrigation techniques and soil conservation, ABC - Crop diversification, irrigation techniques and soil conservation.

Source: survey data, 2013

\subsubsection{Crop Diversification}

In recent years, most of the coffee growers have been faced with the simultaneous risks of drought, abnormal rainfall and pest and disease outbreak coupled with the falling coffee price in the world market. They developed their coffee farms under the intercropping coffee with other crops such as fruit trees with banana, mango, avocado, soursop, rambutan; black pepper or shading trees to reduce the external and internal risks in production and stabilizing income. However, the size of intercropping currently was not large enough to make significant contributions to farmers' income compared with the dominant coffee tree. These crops planted as the boundary for the coffee garden for windbreak purpose, planted at uproot coffee areas with low yield, pests and diseases destroyed and home consumption.

There were $59.3 \%$ and $34.6 \%$ farmers who adapted to crop diversification, asserted that crop diversification adaptation had the moderately effectiveness (4) and 
neutral (3) scales respectively. By contrast, 6.1\% farmers responded that it was a moderately ineffective (2) option because of the capital and knowledge requirements in investing and controlling diseases for other crops, especially black pepper. The neutral and moderately effectiveness evaluation were explained that crop diversification reduced the risk of income loss as a result of climate conditions, but was not considered highly effective because changes in temperature and aberrant rainfall still impacted on some crop varieties, likely leading to some income loss. In addition, some farmers claimed that plantation shading trees with inappropriate density and techniques in the coffee gardens could obstruct the coffee's photosynthesis resulting in the adverse quality of coffee.

In terms of economic efficiency, crop diversification was moderately effective (4) by $59.3 \%$ and neutral (3) with $29.6 \%$ of respondents as the benefits of reduced income loss were expected to exceed the costs of implementation when the pressure of climate conditions took in place in recent years. They claimed that there were some additional costs expected with growing a wider variety of crops, including the possible additional farm equipment for planting, harvesting, storing, labor costs or disease control which were dependent on the nature of the change in cropping practices. There were $11.1 \%$ responded that it was a moderately ineffective (2) adaptation.

However, it was considered as a very flexible (5) adaptation option with $8.6 \%$ and $80.2 \%$ with moderately flexible (4) by giving its potential for yield production under a variety of climate conditions. $11.1 \%$ respondents answered with neutral (3).

There were $59.3 \%$ of farmers answered that this adaptation had moderate implement-ability (4). About $40.7 \%$ responded that it had neither high nor low farmer implement-ability (3) given current social and cultural norms. They were discouraged by the potential complexity of the practice, the need to change established practices, attitudes and norms and additional equipment or contracting, the potential demands on time, knowledge, resources for production and marketing. Moreover, crop diversification as an adaptation was difficult to implement for some farmers who lacked training, skills or investment.

In independent benefits dimension, $86.4 \%$ of farmers responded that crop diversification had moderately independent benefits (4) and $13.6 \%$ with neutral (3) because in addition to reducing the risks associated with changing temperature and rainfall, it reduced risks associated with improvement in soil fertility and reductions in pesticide use through improvements in natural pest resistance.

\subsubsection{Irrigation Techniques}

The irrigation technique which farmers applied included the basal technique, sprinklers system from up to down and saving water irrigation. The coffee farmers claimed that, the implementation of irrigation was a predominant adaptation option for the purpose of improving productivity and reducing risk of income loss, due to recurring drought and rainfall delay. There were $44 \%$ farmers asserted that implementation of irrigation was a very effective (5) adaptation option and moderately effective (4) comprised 54\%. It allowed for the artificial application of moisture during times of stress, maintained and enhanced crop yields relative to climate conditions. The irrigation application helped to meet water demand, improve quality and enhance coffee yield. Only 2\% of farmers responded with moderately ineffective (2) because of unavailability water.

Under economic efficiency aspect, irrigation technique needed more investment cost such as the purchase of irrigation equipment and on-farm distribution infrastructure, while other costs associated with pumping and water allocation volumes would be incurred, they also contributed in proportion of the total costs of irrigation implementation. Thus, irrigation implementation was considered to be very effective (5) with $25 \%$, moderately economically efficient (4) with $73 \%$ and $2 \%$ responding moderately ineffective (2).

Implementation of irrigated agriculture for coffee was considered moderately flexible (4) accounted for $79 \%$. When water was available for irrigation, the adaptation was considered under flexible a variety of moisture constraints over a five-year period. However, given the uncertainties in predicting local and regional changes in precipitation, evaporation and the amount of available soil moisture, implementation of an irrigation system was moderately flexible (2) adaptation strategy with $21 \%$ due to repercussions of water availability and access to irrigation practices.

In terms of farmer implement-ability of irrigation techniques adaptation, there were $17 \%$ of farmers implied very implement-ability (5), moderately implement-ability (4) with 50\% and neutral (3) took up 33\%. Application irrigation technique for their farm required substantial investment in time to learn new skills related to irrigation management given the specific soil and land characteristics of the farm property, the nature of the crop types intended to be irrigated, fertilizer application techniques and credit capacity as well. On the other hand, irrigation as an adaptation need little additional learning and effort, especially if farmers had irrigated in the past such as other farm properties and adjacent fields.

Implementation of irrigation had high independent benefits (5) with responses comprising 77\%. The remained $23 \%$ presented with moderately independent benefits (4). Irrigation enhanced the productivity of many crops irrespective of climate pressure and increases the moisture of soils.

\subsubsection{Soil Conservation}

Overuse of chemicals, groundwater level decrease and increase in evapotranspiration would magnify the vulnerability of coffee plantations to climate pressure. Thus, approaches to enhance the resilience of soils such as organic fertilization, planting trees and bushes or legumes that helped to prevent from soil erosion, enhancement of water storage capacity of the soils should be identified and implemented early enough to avoid serious damages and yield loss. The survey result indicated that there were 91 farmers adopted soil conservation for dealing with climate pressure.

Under effectiveness dimension, $49.5 \%$ of farmers considered soil conservation as a moderately effective adaptation (4) and neutral (3) with 50.5\%. They explained that it promoted in soil fertility, enhanced carbon sequestration, reduced potential for wind and water erosion. This also helped to improve the yield of coffee. 
However, lack of knowledge and appropriate technique in fertilizer application caused the adverse effects on soil fertility and quality of coffee.

Soil conservation provided and maintained an optimum condition of the root-zone to maximum possible depth for coffee roots to function more effectively and without hindrance by capturing high amounts of desired plant nutrients and water passes down to the groundwater and stream flow, not over the surface as runoff. There were 49.5\% respondents claimed moderately effective (4) for term of economic efficiency and $43.9 \%$ answered in neutral scale (3). Soil conservation enhanced soil fertility leading to improve and increase coffee yield. But this also spent more cost for kind of fertilizer, which had high quality and more labor cost as well. Only 6.6\% noted in moderate ineffective (2) scale. They mentioned enhancement coffee productivity and soil moistures thank to irrigation techniques rather than soil conservation.

Dimension of flexibility and implement-ability was evaluated moderate level (4) comprised $49 \%$ respectively. The remaining percent responded with neutral (3). Meanwhile, 58\% farmers considered this adaptation had moderately independent benefits (4) and neutral (3) constituted $42 \%$.

From the survey results about farmers' different scales for each adaptation under various criteria, the study used the unity based normalization tool in order to normalize the original values into the range in $[0,1]$. The scales for the groups who adapted to two and three adaptation options were defined by calculating the average values of scales that they answered for each adaptation. The weight method was also employed to compute the weighted scale for each adaptation option. The result of Table 3 presented how each adaptation performed for each criterion. The finding deposed that crop diversification adaptation had high weight on flexibility. Implementation of irrigation was evaluated high weight scale on effectiveness, economic efficiency and independent benefits. Meanwhile, the weight of scales of economic efficiency, flexibility and implement-ability for soil conservation adaptation were the same. The weighted scale of effectiveness and economic efficiency of the groups who adjusted to crop diversification and irrigation techniques, irrigation techniques and soil conservation and three of the options were higher than the group of crop diversification and soil conservation. In addition, the adaptation groups which involved in the irrigation technique were evaluated strongly in all of criteria. This expressed that irrigation techniques played an essential role for coffee production and appropriately responded to pressure of temperature and rainfall changes.

Table 3. Criteria Weighted Scale and Selected Farmer Adaptations

\begin{tabular}{|c|c|c|c|c|c|c|c|}
\hline \multirow{2}{*}{ Criterion } & \multicolumn{7}{|c|}{ Reduce risk of losses due to climate pressure } \\
\hline & A & $\mathrm{B}$ & $\mathrm{C}$ & $\mathrm{AB}$ & $\mathrm{AC}$ & $\mathrm{BC}$ & $\mathrm{ABC}$ \\
\hline Effectiveness & 0.65 & 0.95 & 0.6 & 0.86 & 0.59 & 0.8 & 0.83 \\
\hline Economic efficiency & 0.71 & 0.94 & 0.56 & 0.86 & 0.62 & 0.75 & 0.74 \\
\hline Flexibility & 0.96 & 0.71 & 0.56 & 0.84 & 0.65 & 0.64 & 0.74 \\
\hline Implement-ability & 0.73 & 0.62 & 0.56 & 0.75 & 0.63 & 0.59 & 0.64 \\
\hline Independent benefits & 0.73 & 0.92 & 0.6 & 0.83 & 0.68 & 0.76 & 0.75 \\
\hline
\end{tabular}

Note: A - Crop diversification, B - Irrigation techniques, C - Soil conservation, AB - Crop diversification and irrigation techniques, AC - Crop diversification and soil conservation, BC - Irrigation techniques and soil conservation, ABC - Crop diversification, irrigation techniques and soil conservation

Source: survey data, 2013

Using the weighted sum method, the rank of the adaptation options was presented in Table 4. Two criteria related to effectiveness and economic efficiency had high weight in adaptation evaluation. The different weights evaluated the relative overall merit of adaptation options.

The finding indicated that the groups who adapted to irrigation techniques, crop diversification and irrigation techniques had the highest sum of weight while the adaptation groups of soil conservation, crop diversification and soil conservation had the lowest weight.

Table 4. Sum of Weighted Scale of Adaptation Evaluation

\begin{tabular}{|c|c|c|c|c|c|c|c|}
\hline \multirow{2}{*}{ Criterion } & \multicolumn{7}{|c|}{ Reduce risk of losses due to climate pressure } \\
\hline & A & B & $\mathrm{C}$ & $\mathrm{AB}$ & AC & $\mathrm{BC}$ & $\mathrm{ABC}$ \\
\hline Effectiveness & 0.62 & 0.90 & 0.57 & 0.82 & 0.56 & 0.76 & 0.79 \\
\hline Economic efficiency & 0.70 & 0.92 & 0.55 & 0.84 & 0.61 & 0.74 & 0.73 \\
\hline Flexibility & 0.56 & 0.41 & 0.32 & 0.49 & 0.38 & 0.37 & 0.43 \\
\hline Implement-ability & 0.47 & 0.40 & 0.36 & 0.48 & 0.40 & 0.38 & 0.41 \\
\hline Independent benefits & 0.42 & 0.53 & 0.35 & 0.48 & 0.39 & 0.44 & 0.44 \\
\hline Sum & 2.76 & 3.17 & 2.15 & 3.11 & 2.34 & 2.68 & 2.79 \\
\hline
\end{tabular}

Note: A - Crop diversification, B - Irrigation techniques, C - Soil conservation, AB - Crop diversification and irrigation techniques, AC - Crop diversification and soil conservation, BC - Irrigation techniques and soil conservation, ABC - Crop diversification, irrigation techniques and soil conservation

Source: survey data, 2013

From the result of Table 4, using the equal interval scale method defined the ordinal three categories of adaptation options (Table 5).

$$
\text { Interval scale }=\frac{3.17-2.15}{3}=0.34
$$

The Table 5 result revealed that in the total of 176 coffee farmers, the low adaptation level, which had the weighted sum from 2.15 to 2.49 , constituted $26.14 \%$. The weighted sum which ranged between 2.50 and 2.84 was considered as the medium adaptation group comprised 
42.61\% while the high adaptation was evaluated with equal or over 2.85 of weighted sum and took up $31.25 \%$.

Table 5. Level of Adaptation Options

Level of adaptation

$$
\text { Low (26.14\%) }
$$

Medium (42.61\%)

$2.50-2.84$

High (31.25\%)

$\geq 2.85$

Source: survey data, 2013

Even though a large number of farmers interviewed noticed changes in temperature and rainfall and selected the adaptation options for their farm, the coffee growers have still faced to difficulties in practicing the adaptations. More than 80 percent of farmers cited lack of access to credit for undertaking the remedial actions. Around 35.80 percentage of farmers designed lack of knowledge of appropriate adaptation measures as barriers to adaptations. There was 28.41 percent of respondents also cited a shortage of labor in adaptation undertake (Figure 1)

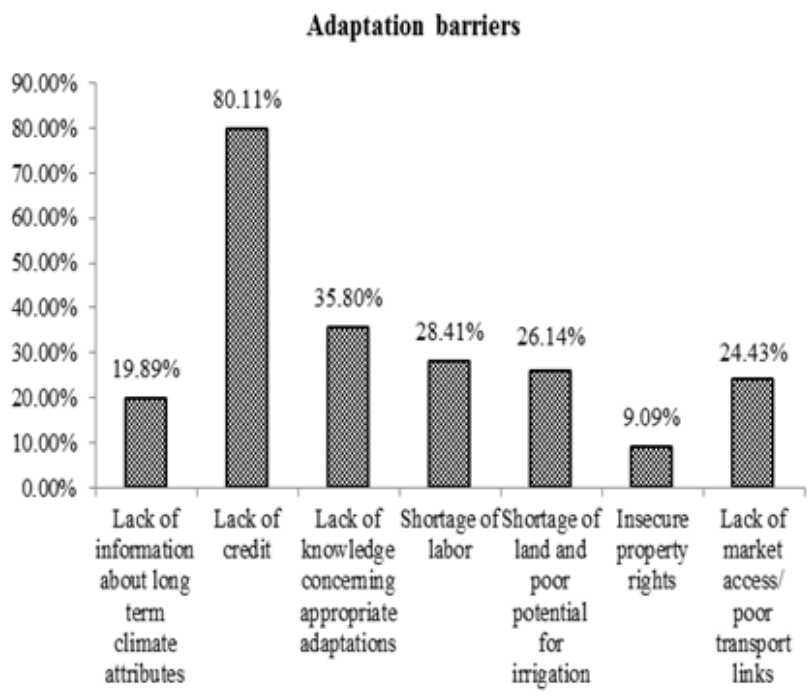

Figure 1. Adaptation barriers (\% of the respondents) Source: Survey data, 2013

\subsection{Factors Influencing the Farmers' Adaptation Level}

The Table 6 noticed that the estimated coefficients for education, coffee growing experience, coffee farming size, coffee income, non-coffee income, access to credit, access to climate information, access to extension services and irrigation option had statistically significant influence on level of adaptation dealing with climate pressure. Only age and gender were insignificant.

The R-squared of model represented the statistical explanatory variables could explain about 44.5 percent of the variation level of farmers' adaptation. The likelihood ratio statistics as indicated by $\chi^{2}$ statistics were highly significant $(\mathrm{P}<0.00000)$, suggesting the model had $\mathrm{a}$ strong explanatory power or high goodness of fit.

The value of threshold parameter explained that, subjects that had a value of 2.43 or less on the underlying latent variable would be classified as moderate adaptation. And the subjects that had a value more than 2.43 on the underlying latent variable would be classified as high adaptation option.

Education of head of household was negatively contributed to levels of low and moderate adaptation while it was positively affected to level of high adaptation. The coefficient value presented that a one unit increase in education would result in a 0.164 unit increase in the ordered log-odds of being in a higher adaptation category while the other variables in the model were held constant. The numbers of schooling years of the head of household increased the probability of adapting to climate pressure. A unit increase in the number of years of schooling would result in a 0.038 percent increase in the probability of choosing high adaptation at significance level $1 \%$.

The coffee growing experience had a positive relationship for choosing high adaptation options and a contrary, the negative effect to low and moderate adaptation. One unit increase in coffee growing experience would lead to a 0.047 unit increase in the ordered log-odds of being in a higher adaptation category while the other variables in the model were held constant. Farmers' coffee growing experience increased one year, the probability of choosing high adaptation increased 0.011 percent at $5 \%$ level.

Table 6. The Estimation of Coffee Farmers' Adaptation on Climate Pressure

\begin{tabular}{|c|c|c|c|c|c|}
\hline \multirow{2}{*}{ Variables } & \multicolumn{2}{|c|}{ Ordered logit model } & \multicolumn{3}{|c|}{ Marginal effects } \\
\hline & Coefficient & S.E & Low & Moderate & High \\
\hline Constant & -2.062 & 0.733 & 0.000 & 0.000 & 0.000 \\
\hline Age (years) & -0.016 & 0.013 & 0.003 & 0.001 & -0.004 \\
\hline Gender ( 1 for female, 0 for male) & -0.291 & 0.237 & 0.042 & 0.029 & -0.071 \\
\hline Education (years) & $0.164 * * *$ & 0.057 & -0.026 & -0.012 & 0.038 \\
\hline CoffeeGrowing Experience (years) & $0.047 * *$ & 0.02 & -0.007 & -0.003 & 0.011 \\
\hline Coffee farming size (hectare) & $0.116^{*}$ & 0.274 & -0.008 & 0.018 & 0.026 \\
\hline Coffee income (1,000,000 VND) & $0.012 * *$ & 0.255 & -0.001 & -0.001 & 0.002 \\
\hline Non-coffee income $(1,000,000 \mathrm{VND})$ & $0.004^{*}$ & 0.002 & -0.001 & -0.000 & 0.001 \\
\hline Access to credit ( 1 for accessing, 0 for otherwise) & $0.637 * *$ & 0.305 & -0.077 & -0.097 & 0.174 \\
\hline Access to climate information ( 1 for accessing, 0 for otherwise) & $0.765 * * *$ & 0.282 & -0.152 & 0.009 & 0.143 \\
\hline Access to extension service ( 1 for accessing, 0 for otherwise) & $0.638 * *$ & 0.254 & -0.106 & -0.035 & 0.141 \\
\hline Irrigation option (1 for irrigation option, 0 for otherwise) & $0.977^{* * *}$ & 0.294 & -0.159 & -0.063 & 0.221 \\
\hline Threshold parameter & 2.43 & 0.272 & & & \\
\hline \multicolumn{6}{|c|}{$\begin{array}{c}\text { Number of observations }=176, \text { McFadden Pseudo R-squared }=44.5 \\
\text { Log likelihood function }=-105.27, \text { Restricted log likelihood }=-189.67 \\
\text { Chi squared }=168.81, \text { Prob }[\text { ChiSqd }>\text { value }]=.000000\end{array}$} \\
\hline
\end{tabular}

Note: *, **, *** Statistically significant at $10 \%, 5 \%, 1 \%$

Source: Survey data, 2013

The coffee farming size: One unit increase in coffee farming size resulted in a 0.116 unit increase in the ordered log-odds of being in a higher adaptation category while the other variables in the model were held constant. 
The value of marginal effect indicated that a unit increased in the coffee farming size made increasing the probability in high adaptation 0.026 percent at $10 \%$ level.

The coffee income: One unit increase in coffee income would lead to a 0.012 unit increase in the ordered log-odds of being in a higher adaptation category while the other variables in the model were held constant. The coffee income of the households surveyed had a positive and significant impact on high adaptation level. A unit increased in the coffee income resulted in increasing the probability in high adaptation 0.002 percent at 5\% level. Farmers, who had higher income, increasing more time for irrigation, building the big tanks to contain storage water as well when raining and apply sprinkler techniques to save water and labor hiring also. However, rising irrigation time spent more cost for fuel and labor. Irrigation technique was limited by requiring abundant water availability and more capital investment. It also required substantial investment in time for farmers learn new skills related to irrigation management and changing in technology as well.

Non-coffee income: The non-coffee income had a positive effect on choosing high adaptation options. One unit increase in non-coffee income would lead to a 0.004 unit increase in the ordered log-odds of being in a higher adaptation category while the other variables in the model were held constant. The marginal effect value highlighted that a unit increased in non-coffee income led to increasing in the probability of level of adaptation with 0.001 percent at $10 \%$ level.

Access to credit: The farmers, who accessed to credit, had more chance or money to invest for high adaptation option. The ordered log-odds for accessing to credit being in a higher adaptation category was 0.637 higher than no access when the other variables in the model were held constant. The probability in adapting high adaptation strategies was higher 0.174 percent than otherwise at $5 \%$ level.

Access to climate information: The ordered log-odds for access to climate information in higher adaptation strategy was 0.765 higher than no access. The farmers, who accessed to climate information or had more information, had a higher probability in high adaptation with 0.143 percent than otherwise at $1 \%$ level.

Access to extension services: the result was in line with various studies in developing countries that report a positive relationship between access to information and the adoption behavior of farmers (Yirga, 2007). The farmers, who had more information, training by extension service programs, had higher the ordered log-odds with 0.638 and higher probability with 0.141 percent in choosing high adaptation strategy at 5\% level.

Irrigation option: The farming households who had irrigation option, the ordered log-odd in a higher adaptation option was 0.977 higher than no irrigation option when the other variables in the model were held constant. The probability in choosing high adaptation was higher than those no option with 0.221 percent at $1 \%$ level.

The insignificant of age was explained that the coffee tree was a perennial crop that required more experience and knowledge for taking care and improving new techniques in processing of coffee cultivation. It hinged on the coffee growers' experience, education or income for investment and development of coffee rather than you were elders.

The result of negative relationship and insignificance between gender and adaptation levels explained that female' probability for selecting adaptation options to climate pressure was lower than male. Through the survey, the women appeared more likely than men to rely on neighbors for information, whereas men appeared more likely than women to hinge on traditional knowledge. Male and female farmers had identical perceptions of temperature and precipitation trends and over time these perceptions matched well with the climate records which shown an increase in climate condition over the past ten years. However, the gender differences in access to institutions, information and acclimatize to climate variability reflected differences in male and female's education levels and literacy as well as culturally defined roles in decision making and division of labor. The role of women in making decision was still weak and did not interact with extension agents or training programs. As a result would be less likely to report having the choice to adaptation strategies.

The result of Table 7 showed that there were more actual farmers with low adaptation level than predicted, 46 of actual versus 38 of predicted. Similarly, there were more actual farmers with moderate adaptation level than predicted, 75 of actual versus 54 of predicted and 55 of actual versus 41 of predicted for high adaptation level. The high predicted percent concluded that the model was more appropriate in explaining the variation of dependent variables when the explanatory elements changed.

Table 7. The Accuracy of Ordered Logit Model

\begin{tabular}{|c|c|c|c|c|}
\hline \multirow{2}{*}{ Level of adaptation } & \multicolumn{3}{|c|}{ Predicted outcome } & \multirow{2}{*}{ Actual Outcome } \\
\hline & Low & Moderate & High & \\
\hline Low & 38 & 8 & 0 & 46 \\
\hline Moderate & 8 & 54 & 13 & 75 \\
\hline High & 0 & 14 & 41 & 55 \\
\hline Percent (Actual over predicted) & 82.61 & 72 & 74.55 & \\
\hline
\end{tabular}

\section{Conclusions}

To investigate the farmers' motivation and adaptation strategies for acclimatizing to climate change, it is essential to examine how farmers evaluate their private adaptive measures and influential factors on their adaptation level. The adaptation options, which were selected for coping with climate pressure on their coffee farming included soil conservation, crop diversification and irrigation techniques. Through the Multi-Criteria Evaluation tools with five criteria, including effectiveness, economic efficiency, flexible, farmer implement ability and independent benefits, the ordinal adaptation strategies were ranged under the low, moderate and high adaptation levels respectively. The result of the multiple criteria evaluation indicated that the adaptation groups related to applying irrigation techniques such as the sprinkler 
irrigation system from up to down, water saving irrigation and crop diversification or soil conservation parallel were assessed strongly rather than responding to only the basal irrigation method, crop diversification or soil conservation. The application of the water saving irrigation technique is not still popular and extensive for the whole of coffee farm scales. In addition, the findings of regression model also revealed that the education level of the household head was strongly and positively associated with all three adaptation levels. Extension for crop production, access to information to climate pressure and access to credit enhanced adaptation to climate variability. However, most of the adaptation measures implemented in the study area are reactive rather than proactive, autonomous rather than well-planned approaches under level of private agents. Therefore, the adaptation for coping with climate pressure and the process of agricultural development are considered to be amenable to policy interventions, joining activity and consultancy of technology scientists and experts. There is a need by agricultural economists to design strategies that could help the farmers or rural communities' responses effectively to changes in temperature and rainfall or global warming in general.

\section{Acknowledgement}

The paper is part of a Master research at Center for Agricultural Resource System Research, Faculty of Agriculture, University of Chiang Mai, Thailand. It is conducted under the spornor of IDRC-SEARCA. We are also very grateful to Department of Agricultural and Rural Development of Daklak province, Agricultural Department and Meteorological Station of Ea H'leo District for their help, information provision and support in organising farmer interviews. We would like to thank four undergraduate students of Nong Lam University, local guides and farm households in the Ea H'leo District in helping and supporting our interviews during June and August 2013.

\section{References}

[1] UNFCC, O.A. Climate Change-Impact, Vulnerabilities and Adaptation in Developing Countries http://maps.grida.no/go/graphic/climate_change_processes_charac teristics_and_treats [20 March 2013], 2007.

[2] Tuan, B., "Implementation of Coffee Value Chian for Ethnic Minorities, Rural Development in Dak Lak, Daklak, 2012.

[3] Haggar, J., "Impacts of Climate Change in the Pilot Country Vietnam the Coffee and Climate Initiative", in Coffee and Climate Change, 2011.

[4] Haggar, J., Coffee and Climate Change Impacts and Options for Adaptation In Brazil, Guatemala, Tanzania and Vietnam, Climate Change, Agriculture and Natural Resource, 2012.

[5] Ea H'leo Agricultural Department, The Situation of The Irrigational Works in the Ea H'leo District, [Online]. Available: http://sonongnghiepdaklak.gov.vn/t.aspx?id=9950, 2013.

[6] Haggar, J., Coffee and Climate Change Impacts and Options for Adaptation in Brazil, Guatemala, Tanzania and Vietnam, in Climate Change, Agriculture and Natural Resource, 2012.

[7] Ea H'leo Agricultural Department, The status of coffee production in Ea H'leo district in 2013, Ea H'leo, Department of Agriculture and Rural Development, Daklak, 2013.

[8] Gay, C., Estrada, F., Conde C., Eakin H. and Villers, L., "Potential Impacts of Climate Change on Agriculture: A Case of Study of Coffee Production in Veracruz, Mexico", Climatic Change, 79, 259-288, 2006
[9] Smith, J.B. and Lenhart, S.S., "Climate Change Adaptation Policy Options", Climate Research, 6, 193-201, 1996.

[10] Klein, R.J.T. and Tol, R.S.J., Adaptation to Climate Change: Options and Technologies, Vrije Universiteit, Amsterdam, 1997.

[11] Yamane, Statistics an Introductory Analysis, 2nd Ed, New York, Harper and Row, 1967.

[12] RDDL, Daklak Develop Coffee Sustainability and Project Region. [Online] Available: http://www.rddldaklak.org/publications/rddl_mandatory_viet_2586032.html [22 May 2013], 2013.

[13] Titus, J.G., The Costs of Climate Change to the United States, In Mjumdar, S.K., Kallstein, L.s., Yarnal, B.M., Miller, E.W. and Rosenfiel, L.M. (Eds.), Global Climate Change: Implications, Challenges and Mitigation Measures, The Pennsylvania Academy of Science Publications, Easton, 384-409, 1992.

[14] Carter, T.R., Harasawa, H. and Nishioka, S., IPCC Technical Guidelines for Assessing Climate Change Impacts and Adaptations, Department of Geography, University College London, London, 1994.

[15] Smith, J.B., Using a Decision Matrix to Assess Climate Change Adaptation, In Smith, J.B., Bhatti, N., Menzhulin, G., Benioff, R., Budyko, M.I., Campos, M., Jallow, B. and Rijsberman, F. (Eds.), Adapting to Climate Change: An International Perspective, Springer, New York, 68-79, $1996 \mathrm{~b}$.

[16] Fankhauser, S., The Potential Costs of Climate Change Adaptation, in Smith, J.B., Bhatti, N., Menzhulin, G., Bennioff, R. Budyko, M., Campos, M., Jallow, B. and Rijsberman, F. (Eds.), Adapting to Climate Change: An international perspective, Springer, New York, 80-96, 1996.

[17] Benioff, B., Guil, S. and Lee, J., Assessment of Adaptation Policy Options, In Benioff, B., Guill, S. and Lee, J. (Eds.), Vulnerability and Adaptation Assessments: An International Handbook, Kluwer Academic Publishers, Dordrecht, 7.1-7.8, 1996.

[18] Carter, T.R., "Assessing Climate Change Adaptations: The IPCC Guidelines”, In Smith, J.G., Bhatti, N., Menzhulin, G., Benioff, R., Budyko, M. I., Campos, M., Jallow, B., Rijsberman, F. and Dixon, R.K. (Eds.), Adapting to Climate Change: An International Perspective Springer, New York, 27-43, 1996.

[19] Hobbs, B.F., Chankong, V., Hamadeh, W. and Stakhiv, E.Z, "Does Choice of Multicriteria Method Matter? An Experiemtn in Water Resources Planning", Water Resources Research, 28(7), 1767-1799, 1992.

[20] Munda, G., Nijkamp, P. and Rietveld, P., "Qualitative Multicriteria Evaluation for Environmental Management", Ecological Economics, 10, 97-112. 1994.

[21] De Bruin, K., Dellink, R.B., Ruijs, A., Bolwidt, L., Van Buuren, A., Graveland, J., De Groot, R.S., Kuikman, P.J., Reinhard, S., Roetter, R.P., Tassone, V.C., Erhagen, A. and van Ierland, E.C.. , "Adapting to Climate Change in the Netherlands: An Inventory of Climate Adaptation Options and Ranking of Alternatives", Climatic Change, 95 (1-2), 23-45, 2009.

[22] Mizina, S.V., Smith, J.B., Gossen, E., Spiecker, K.F. and Witkowski, S.L., "An Evaluation of Adaptation Options for Climate Change Impacts on Agriculture in Kazakhstan", Mitigation and Adaptation Strategies for Global Change, (4), 2541, 1999.

[23] Fankhauser, S. and Tol, R.S.J., "The Social Costs of Climate Change: The IPCC Second Assessment Report and Beyond", Mitigation and Adaptation Strategies for Global Change, 1, 385403, 1997.

[24] Brklacich, M., McNabb, D., Bryant, C. and Dumanski, J. Adaptability of Agriculture Systems to Global Climate Change: A Renfrew County, Ontario, Canada Pilot Study, In Ilbery, B. Chiotti, Q. and Rickard, T. (Eds.), Agricultural Restructuring and Sustainability: A geographical perspective, CAB International, Wallingford, 351-364, 1997b.

[25] Smithers, J. and Smit, B., "Agricultural System Response to Environmental Stress”, In Ilbery, B., Chiotti, Q. and Rickard, T. (Eds.), Agricultural Restructuring and Sustainability, 167-183, 1997.

[26] Arthanari, T.S. and Dodge, Y., Mathematical Programming in Statistics, Wiley, 1993.

[27] Rosenberg, N.J., "Adaptation of Agriculture to Climate Change", Climatic Change, 21, 385-405, 1992.

[28] Maclaren, V., Multicriteria Evaluation Methods, In V. Maclaren and Whitney J. (Eds.), New Directions in Environmental Impact Assessment, Methuen, Toronto, 1985. 
[29] Marler, T. and Arora, J. S., "The Weighted Sum Method for Multi-Objective Optimization: New Insights", Struct Multidisc Optim, 41, 853-862., 2010.

[30] Steven, S.S., "On the Theory of Scales of Measurement". Science, 103 (2684), 677-680, 1946

[31] Green, Econometric Analysis, 4th ed, Prentice Hall Upper Saddle River, New Jersey: Prentice-Hall, 2000.

[32] Asfaw, A. and Admassie, A., "The role of education on the adoption of chemical fertilizer under different socioeconomic environments in Ethiopia”, Agricultural Economic, 30 (3), 215228, 2004.

[33] Tenge, J., De Graaff, E. and Hella, J.P., "Social and economic factors affecting the adoption of soil and water conservation in West Usambara highlands, Tanzania", Land Degradation and Development, 15 (2), 99-114, 2004.

[34] Norris, E. and Batie, S., "Virginia farmers' soil conservation decisions: An application of Tobit analysis", Southern Journal of Agricultural Economics, 19 (1), 89-97, 1987.

[35] Igoden, C., Ohoji, P., and Ekpare, J., "Factors associated with the adoption of recommended practices for maize production in the Lake Basin of Nigeria", Agricultural Administration and Extension, 29 (2), 149-156, 1990.
[36] Maddison, D., The perception of and adaptation to climate change in Africa, CEEPA. Discussion Paper No. 10, Centre for Environmental Economics and Policy in Africa, Pretoria, South Africa: University of Pretoria, 2006.

[37] Nhemachena, C. and Hassan, R., Micro-level analysis of farmers' adaptation to climate change in Southern Africa, IFPRI Discussion Paper No. 00714, International Food Policy Research Institute, Washington, D.C, 2007.

[38] Knowler, D. and Bradshaw, B., "Farmers' Adoption of Conservation Agriculture: A Review and Synthesis of Recent Research", Food Policy, 32 (1), 25-48, 2007.

[39] Franzel, S., "Socioeconomic Factors Affecting the Adoption Potential of Improved Tree Fallows in Africa", Agroforestry Systems, 47 (1-3), 305-321, 1999.

[40] CIMMYT (International Maize and Wheat Improvement Center), The Adoption of Agricultural Technology: A Guide for Survey Design, Economics Program, Mexico City, Mexico, 1993.

[41] Yirga, C.T., The dynamics of soil degradation and incentives for optimal management in Central Highlands of Ethiopia, $\mathrm{PhD}$ thesis, Department of Agricultural Economics, Extension, and Rural Development. University of Pretoria, South Africa, 2007.

[42] Pattanayak, S.K., Mercer, D.E., Sills E. and Jui-Chen, Y., "Taking Stock of Agroforestry Adoption Studies”, Agroforestry System, 57 (3), 173-186, 2003 